and other organs. As pancreatic expression of IFN- $\gamma$ reduced myocarditis, the authors considered two possible pathogenic mechanisms of autoimmune myocarditis: a virus-induced immune response caused by damaged myocytes, or 'molecular mimicry' between epitopes shared by the virus and antigens of the heart. Although autoantibodies against cardiac myosin were present in the transgenic CB3-infected NOD mice as well as in the non-transgenic infected NOD mice, the authors concluded that 'molecular mimicry' was not likely to be a mechanism, because cardiac damage induced by CB3 was required to initiate disease, probably by exposing heart antigens (Fig. 1).

Additional studies have approached the same question from a different direction. Comparison of myosin amino-acid sequences reported to be responsible for disease induction ${ }^{9}$ with viral sequences has demonstrated little overlap. In addition, immunization with killed coxsackievirus in complete Freund's adjuvant fails to induce disease. Finally, antibodies induced by immunization with myosin and CB3 do not cross-react with the same proteins ${ }^{10}$. Admittedly, none of these experiments exclude the possibility that 'molecular mimicry' between virion and host is involved in inducing autoimmune disease after viral infection. Nonetheless, several lines of evidence indicate that viral infection can induce cell damage and exposure of host antigens ${ }^{11}$. It is likely that the virus-induced injury causes release of intracellular cardiac proteins (such as myosin) which, through activation of inflammatory cells, could lead to autoimmune myocarditis in genetically susceptible mice.

These findings have direct relevance to the diagnosis and treatment of the many human diseases in which a microbial infection is linked with an autoimmune process. The treatment of autoimmune disease by global immunosuppression is contraindicated for an infectious disease. It is important to distinguish infectious from autoimmune pathology, and to treat each appropriately.

1. Horwitz, M.S. et al. Pancreatic expression of interferon-g protects mice form lethal coxsackievirus B3 infection and subsequent myocarditis. Nature Med. 6, 693-697 (2000).

2. Rose, N.R. \& Mackay, I.R. Molecular mimicry: a critical look at exemplary instances in human diseases. Cell. Mol. Life Sci. 57, 542-551 (2000).

3 Albert, L.J. \& Inman, R.D. Molecular mimicry and autoimmunity. N. Engl. J. Med. 341, 2068-2074 (1999).

4. Evans, C.F., Horwitz, M.S., Hobbs, M.V. \& Oldstone, M.B. Viral infection of transgenic mice expressing a viral protein in oligodendrocytes leads to chronic central nervous system autoimmune disease. J. Exp. Med. 184, 2371-2384 (1996).

5. Pauschinger, M. et al. Enteroviral RNA replication in the myocardium of patients with left ventricular dysfunction and clinically suspected myocarditis. Circulation 99, 889-895 (1999).

6. Huber, S.A. Animal models of human diseaseAutoimmunity in myocarditis: relevance of animal models. Clin. Immunol. Immunopathol. 83, 93-102 (1997)

7. Caforio, A.L., Goldman, J.H., Haven, A.J., Baig, K.M., \& McKenna, W.J. Evidence for autoimmunity to myosin and other heart-specific autoantigens in patients with dilated cardiomyopathy and their relatives. Int. J. Cardiol. 54, 157-163 (1996).

8. Neu, N. et al. Cardiac myosin induces myocarditis in genetically predisposed mice. J. Immunol. 139, 3630-3636 (1987).

9. Hill, S.L. \& Rose, N.R. in The Molecular Pathology of Autoimmune Diseases $2^{\text {nd }}$ ed. (eds. Theofilopoulos. A.N. \& Bona, C.A.) (Gordon and Breach, Langhorne, Pennsylvania, in the press).

10. Neu, N., Craig, S.W., Rose, N.R., Alvarez, F. \& Beisel, K.W. Coxsackievirus induced myocarditis in mice: cardiac myosin autoantibodies do not cross-react with the virus. Clin. Exp. Immunol. 69, 566-574 (1987)

11. Miller, S.D. et al. Persistent infection with Theiler's virus leads to CNS autoimmunity via epitope spreading. Nature Med. 3, 1133-1136 (1997).

\section{Department of Pathology and}

Department of Molecular Microbiology and Immunology

The Johns Hopkins Medical Institutions MMI/Room E5014

Baltimore, Maryland 21205, USA

Email:nrrose@jhsph.edu

\title{
Tying-up tuberculosis
}

Scientists have known for decades that the virulence of many strains of pathogenic bacteria can be correlated with colony morphology. Koch observed more than 100 years ago that the virulence of Mycobacterium tuberculosis (Mtb) is correlated with the formation of braided microscopic bundles, known as 'cording' (visualized by auramine rhodamine fluorescent stain in picture). Bacterial colony structure is determined by cell wall components, but detailed studies of this complex mixture of lipids and glycolipids in Mtb have been limited by a lack of cell surface mutants. Recent advances in Mtb genetics, along with the completion of its genome sequence, have allowed researchers to genetically screen mutants with altered colony morphologies and investigate the involvement of the cell envelope in mycobacterial virulence. In the April issue of Molecular Cell, Glickman et al. reported the identification of a mycobacterial gene, $p c a A$, that is required for cording and

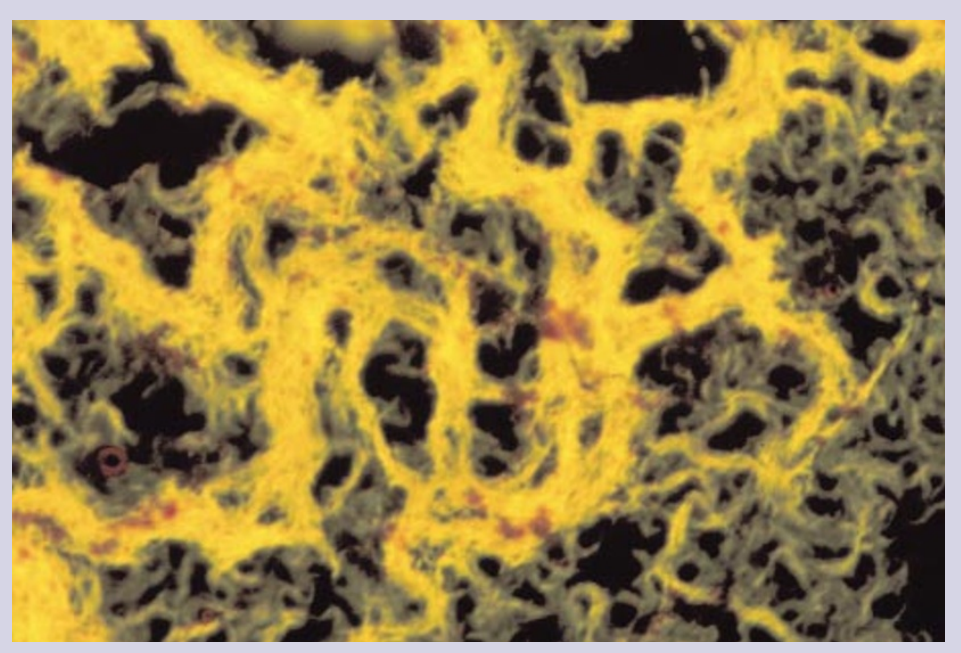
persistent infection. PcaA is a cyclopropane synthase involved in the formation of mycolic acids, modified membrane lipids found in many bacterial species. The authors found that $p c a A$ mutant bacteria initially replicated normally in vivo, but failed to persist within and kill infected mice, indicating that cyclopropanated lipids are required for latent Mtb infections. Mice infected with the pcaA mutant strain did not experience severe pulmonary damage, and had alterations in the composition of inflammatory cell infiltration compared with that of mice infected with wild-type Mtb. "Studies of this type are revealing that lipid and glycolipid molecules are important determinants of Mtb pathogenesis - some lipids are important for initial in vivo replication in the organism, while others are important for long-term persistence," explained Glickman. The authors plan to better characterize the immune response to pcaA and to test the pcaA mutant in formal models of Mtb latency, a condition that affects approximately $32 \%$ of the world's population. As cyclopropanated lipids do not exist in humans, pcaA may also make a good drug target.

Kristine Novak 